\title{
Increased solubility and mobility of tetravalent actinides through silicate colloid formation - An analog study using $\mathrm{Zr-89}$ radiotracer
}

\author{
STEFAN SCHYMURA, ALEXANDER MANSEL AND \\ KARSTEN FRANKE
}

Helmholtz-Zentrum Dresden - Rossendorf e. V. (HZDR)

Presenting Author: s.schymura@hzdr.de

Tetravalent actinides form colloids at near neutral $\mathrm{pH}$ in the presence of silicic acid [1]. This may have significant impact on actinide mobility in the far field of a nuclear waste repository or the case of accidental release of actinides into the environment as silicate is ubiquitous in natural waters and is of special importance in the case of nuclear waste repositories in crystalline rock formations.

We investigated the formation, stability and mobility of such colloids employing the tetravalent actinide analog zirconium and the radiotracer $\mathrm{Zr}-89$. The radiotracer was produced at the inhouse IBA Cyclone 18/9 cyclotron at the HZDR Research Site Leipzig. A foil of metallic yttrium was irradiated with $14.5 \mathrm{MeV}$ protons to trigger the nuclear reaction Y-89(p,n)Zr-89. The target was subsequently dissolved and the radiotracer was isolated via ion chromatography methods using UTEVA resin.

The radiotracer was employed to synthesize zirconium oxyhydroxy silicate colloids that form spontaneously at near neutral $\mathrm{pH}$ at $\mathrm{Si}$ concentrations between $0.5-2 \mathrm{mM}$. In contrast to the $\mathrm{Zr}(\mathrm{O})(\mathrm{OH})_{2}$ particles that precipitate without silicates these colloids remain in stable dispersion. Column experiments in model porous media reveal a potentially massively increased mobility of these colloids due to very low affinities for the geomatrix surface.

The stability of these colloids was further investigated by producing particles from solutions containing various $\mathrm{Zr}$ : $\mathrm{Si}$ ratios. Homoaggregation affinity measurements on these systems by dynamic light scattering reveal an increasing stabilization of the colloids with increasing silica content hinting at (electro)steric stabilization mechanisms due to polymeric silicic acid structures at the colloid surface. Consequently an increased mobility of low concentration tetravalent actinides through colloid formation may occur in environments with silicic acid presence.

[1] Zänker, Weiss, Hennig, Brendler \& Ikeda-Ohno (2016), ChemistryOpen 5, 174-182. 